\title{
LASER SHOCK PROCESSING: \\ AN EMERGING TECHNIQUE FOR THE ENHANCEMENT OF SURFACE PROPERTIES AND FATIGUE LIFE OF HIGH STRENGTH METAL ALLOYS
}

\author{
J.L. Ocaña, J.A. Porro, M. Morales, D. Iordachescu, M. Díaz, L. Ruiz de Lara, C. Correa and A. Gil-Santos \\ Centro Láser UPM. Universidad Politécnica de Madrid \\ Campus Sur UPM. Ctra. de Valencia, km. 7.3. 28031 Madrid. SPAIN
}

\begin{abstract}
:
Profiting by the increasing availability of laser sources delivering intensities above $10^{9} \mathrm{~W} / \mathrm{cm}^{2}$ with pulse energies in the range of several Joules and pulse widths in the range of nanoseconds, laser shock processing (LSP) is being consolidating as an effective technology for the improvement of surface mechanical and corrosion resistance properties of metals and is being developed as a practical process amenable to production engineering. The main acknowledged advantage of the laser shock processing technique consists on its capability of inducing a relatively deep compression residual stresses field into metallic alloy pieces allowing an improved mechanical behaviour, explicitly, the life improvement of the treated specimens against wear, crack growth and stress corrosion cracking. Following a short description of the theoretical/computational and experimental methods developed by the authors for the predictive assessment and experimental implementation of LSP treatments, experimental results on the residual stress profiles and associated surface properties modification successfully reached in typical materials (specifically steels and Al and Ti alloys) under different LSP irradiation conditions are presented
\end{abstract}

Keywords: laser shock processing, laser-plasma interaction, numerical simulation, experimental characterization, residual stresses, mechanical behaviour improvement, fatigue life improvement

\section{Introduction}

Laser Shock Processing (LSP) is consolidating as a competitive alternative technology to classical treatments for the improvement of surface properties of metallic alloys involving the fatigue life of critical components. Specially wear resistance, stress corrosion cracking susceptibility and crack propagation rate seem to be material properties specially improved by LSP treatments [1-4].

On the basis of laser sources able to provide intensities exceeding the $\mathrm{GW} / \mathrm{cm}^{2}$ level, the LSP technology is aimed to be developed from an industrial point of view for the improvement of the fatigue cracking resistance and other surface properties of materials used in the aerospace, nuclear and automotive applications, such as Aluminum and Titanium alloys and different types of stainless steel [5-6]. This confers the LSP technique a clear character of sustainability-supporting technique as far as the whole life cycle of critical components in the referred sectors is considered.
However, as a consequence of the inherent physical complexity of LSP processes, specially stemming on the coexistence of different material phases (including plasma) developing and interacting under the action of the high intensity laser beam, very limited attempts have been developed in the way of full comprehension and predictive assessment of the characteristic physical processes and material transformations with a specific consideration of real material properties (see, for example, references [79]).

This particular situation, not very common in classical high power laser applications, poses the need for the development of comprehensive analysis and prediction tools enabling an integrated comprehension of the physical phenomena developing in the process and their mutual interrelations. Only very few contributions (see, for example, references [10-13] from the authors) have been devoted to this coupled analysis with the aim to correlate laser incidence and interaction material-geometry parameters to final 
thermomechanical effects resulting in treated specimens.

In this paper, after a short description of the theoretical/computational and experimental methods developed by the authors for the predictive assessment and experimental implementation of LSP treatments, experimental results on the residual stress profiles created in $\mathrm{Al}$ and $\mathrm{Ti}$ alloys under different irradiation conditions are presented along with the associate effects on characteristic material surface properties, namely surface roughness and wear resistance. Based on the analysis of these results, experimental guidelines are obtained about the presumable degree of protection provided by the LSP treatment against mechanical failure and the corresponding life extension expectations.

\section{Theoretical process modelling}

\subsection{Model description}

The predictive assessment of the large amount of physical phenomena arising in LSP processes requires a deep understanding of the physics underlying the different phases developing along the laser interaction and is absolutely needed for the reliable development of practical experiments leading to effective material transformations. Such modelling must explicitly consider the formation of a plasma phase, its thermofluiddynamic behaviour under extreme pressure and temperature conditions (typically leading to pressure/shock waves), the propagation of these shock waves into the solid material and the analysis of the behaviour of the material itself under the shockwave energy release (the direct cause for its desired plastic deformation).

The appropriate description of the process requires a three level description providing the adequate interconnection of the data obtained in each phase in a self-consistent way from the physical point of view. This sort of simulation offers the possibility of an independent validation of each model, providing the chance for a modular growing of the physical models implemented complexity.

The referred three-level description includes:

i) Analysis of the plasma electronic population dynamics, including consideration of breakdown phenomenology in dielectric media

ii) Simulation of the hydrodynamic phenomenology arising from plasma expansion between the confinement layer and the base material iii) Analysis of the propagation and induction of permanent structural changes by shock wave evolution in bulk material

A calculational model dealing with these three main aspects of the process modelling in a coupled way has been developed by the authors. The developed calculational model (SHOCKLAS) is integrated by three principal modules, respectively called LSPSIM, HELIOS and HARDSHOCK, and has been conceived for the analysis of the problem of laser shock waves generation and propagation under three different but intercomplementary approaches (see references [10-16]). Fig. 1, shows a schematic representation of the way of coupling of the referred modules.

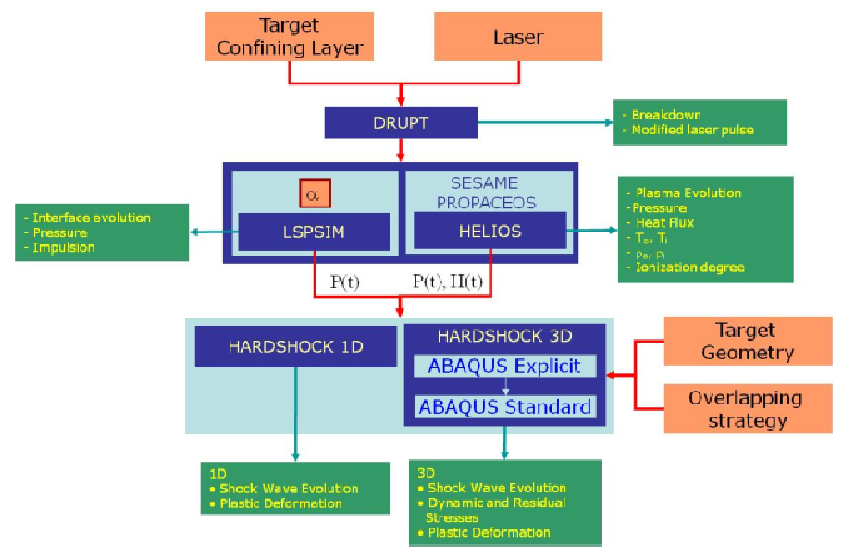

Fig. 1: Scheme of the coupled model (SHOCKLAS) used for the analysis of LSP processes.

\subsection{Numerical model results}

As a sample of the capabilities of the SHOCKLAS calculational system, the analysis of a fundamental issue in the application of the LSP technique to materials susceptible of microstructure changes due to thermal heating-cooling cycles is presented here. Such issue is the evolution of the resulting state of the treated material after the propagation of the shock wave induced by laser interaction. Bearing in mind that the desired effect of the LSP treatment is the induction of permanent compressive residual stress fields of certain intensity and certain depth into the material, the practical consequence arises on the need for a predominantly mechanical plastic deformation (with induction of crystal dislocations) with respect to deformations of thermal origin, normally reversible with time or subsequent heating. The verification of this condition in view of the suitability of the LSP technique for critical applications has also been studied with the aid of the SHOCKLAS calculational 
system, this time involving the coupled capabilities provided by the HELIOS and HARDSHOCK codes.

According to HELIOS results, for typical LSP conditions, the thermal and mechanical pulses applied to the solid target as a consequence of the laser plasma interaction are, in practice, partially uncoupled, so that the characteristic times for propagation of the corresponding thermal and mechanical (shock) waves into the bulk mass are substantially different. However, the generated thermal wave is anyway of a very high intensity and a complete analysis of the problem should involve the evaluation of its effects, with explicit consideration of the different characteristic propagation speeds of both types of waves into the solid material. In Fig. 2, a typical plot of the correlated temporal evolution of both types of waves applied to the solid target is shown.

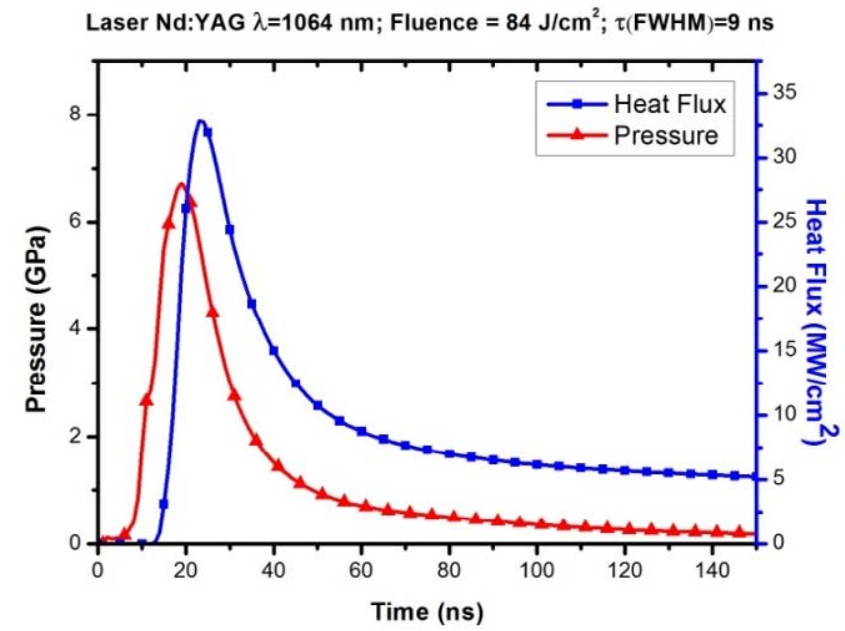

Fig. 2: Comparative temporal evolution of thermal and mechanical waves applied to a solid (Al) target with water confinement in typical LSP conditions.

On the basis of these characteristic applied profiles, the effect on the LSP generated residual stress fields of the combined action of thermal + mechanical effects has been compared to the effects induced by a purely mechanical treatment (i.e. without consideration of any thermal effects) has been studied with the aid of the HARDSHOCK code.

In Fig. 3, the comparison is presented (in terms or residual radial stressed fields along the specimen depth) of results corresponding, on one side, to uncoupled thermal and mechanical analysis and, on the other to a fully coupled thermo-mechanical analysis (see reference [13] for more details).

From the observation of the figure, the clear conclusion can be extracted of the relative importance of thermal effects in points of the material close to the surface, so that, for this region, thermal effects can by no means be neglected, especially in the case of materials especially susceptible to thermal microstructure modification. On the other hand, however, for depths into the material relatively far from the surface, where the protective effect of compressive residual stresses is important from the point of view of material toughness, the effect of the thermal wave generated by laser interaction can be considered as practically extinguished.

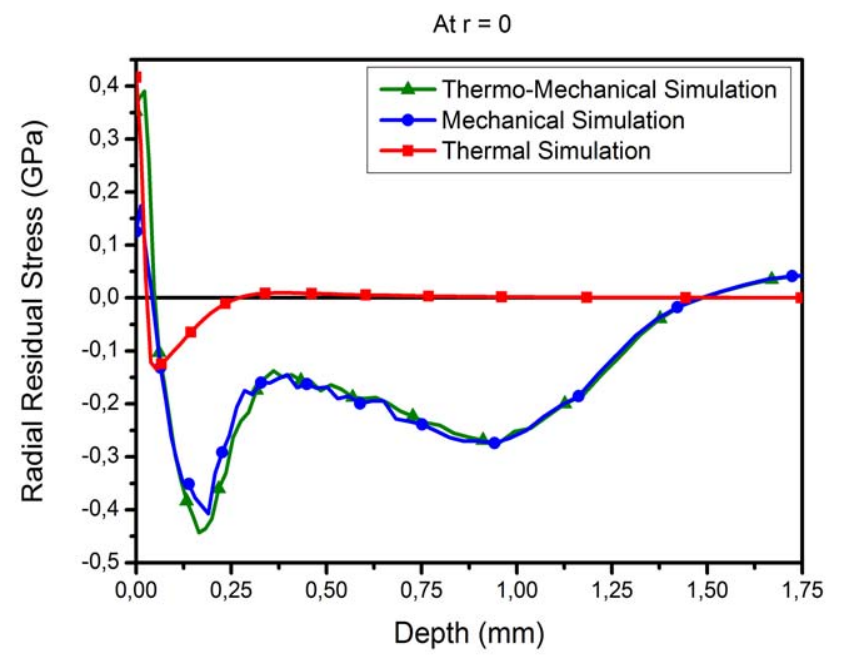

Fig. 3: Relative effects in residual stresses induced in Aluminum due to the thermal and mechanical waves generated by laser interaction in LSP conditions.

However, the most interesting feature of the SHOCKLAS code is its ability to obtain fully 3D profiles of the residual stresses fields obtained in a treated material. In Fig. 4, a sample is presented showing the capability of the code to obtain a full spatial dependence of residual stresses and surface deformations for a given pulse overlapping strategy. In this view the interesting effect can be observed of the homogeneization of the residual stresses fields at the surface level after a minimum level of pulse overlapping factor.

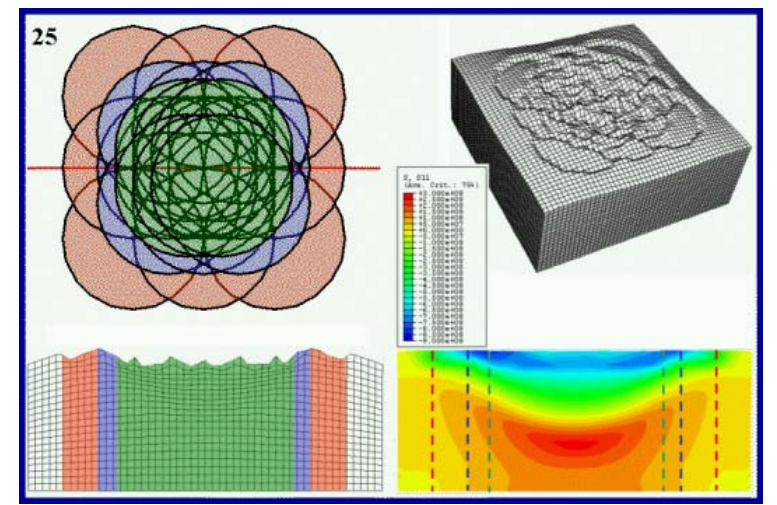

Fig. 4: Sample RS's fields and surface deformation profiles obtained by means of $3 D$ SHOCKLAS code for a typical pulse overlapping strategy. 


\section{Experimental results}

The practical irradiation system used for the experiments reported in this paper is photographically shown in Fig. 5. Using purified water as confining medium, the test piece is fixed on a holder and is driven along $\mathrm{X}$ and $\mathrm{Y}$ directions by means of a computer controlled stage needed for the irradiation of extended areas of material following a pre-defined pulse overlapping strategy. The laser light is conducted to the interaction area by means of a reflecting mirror and a focusing lens (see additionally references [17] for more details).

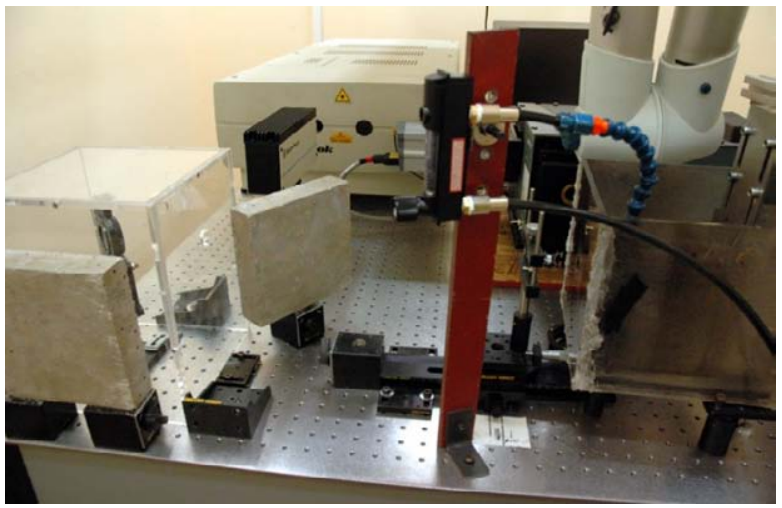

Fig. 5: Photographic view of the LSP irradiation experimental setup used at CLUPM.

The LSP experiments reported in this paper were performed on A12024-T351 and Ti6A14V alloys at $1064 \mathrm{~nm}$ laser wavelength using a Q switched $\mathrm{Nd}$ :YAG laser operating at $10 \mathrm{~Hz}$ and providing 9.4 ns FWHM, 2.0 J pulses. A convergent lens was used to deliver the laser energy over a $1.5 \mathrm{~mm}$ spot diameter. The confining layer was provided by a water jet incident close to the laser interaction zone. No protective coatings were used in the experiments. Besides laser pulse energy and interaction diameter, the main varying experimental parameter is the socalled "overlapping pitch", $d$, a direct measure of the distance between both adjacent laser shots and parallel processing tracks which, in the defined geometry, implies a given pulse density according to the relation:

$\begin{aligned} & \text { Equivalent } \\ & \text { Overlapping } \equiv \mathrm{EOD}=\frac{\mathrm{N}^{\mathrm{o}} \text { of pulses }}{\text { Density }}\end{aligned}=\frac{\frac{\mathrm{x}}{\Delta \mathrm{x}} \frac{\mathrm{y}}{\Delta \mathrm{y}}}{\Delta \mathrm{s}}=\frac{\frac{\mathrm{x}}{\mathrm{d}} \mathrm{d}}{\mathrm{xy}}=\frac{1}{\mathrm{~d}^{2}}$

Under the referred experimental conditions, the effects induced by the LSP treatment on the mechanical properties of two characteristic alloys, namely Al2024-T351 and Ti6Al4V, have been characterized. In particular, the analysis of induced residual stress fields has been accomplished according to their primary significance. Additionally, associated surface roughness and wear resistance have been evaluated. Specimens $8 \mathrm{~mm}$ thick were used for the experiments. Equivalent overlapping densities reported in this paper were, respectively, 900, 1600 and 2500 pulses $/ \mathrm{cm}^{2}$ for A12024-T351 samples and 900, 2500 and 5000 pulses $/ \mathrm{cm}^{2}$ for Ti6Al4V samples.

Residual stress distributions were determined according to the ASTM E837-08 Standard Test Method for Determining Residual Stresses by the Hole Drilling Strain Gage Method [18]. Strain gage rosettes CEA-13-062UM-120 along with a Vishay Measurements ${ }^{\circledR}$ RS-200 milling guide were used. On its side, the wear resistance tests were performed according to the ASTM G99-04 Standard Test Method for Wear Testing with a Pin on Disk Apparatus [19] on a Microtest ${ }^{\circledR}$ MT/30 system. The samples' roughness was characterized by means of a Laser Confocal Microscope Leica® ICM 1000.

\subsection{Residual Stresses Fields}

Figs. 6 and 7 show the depth profiles obtained in the two materials considered for LSP-induced Mohr maximum (minimum in absolute value) residual stresses for three representative values of effective pulse density in each case.

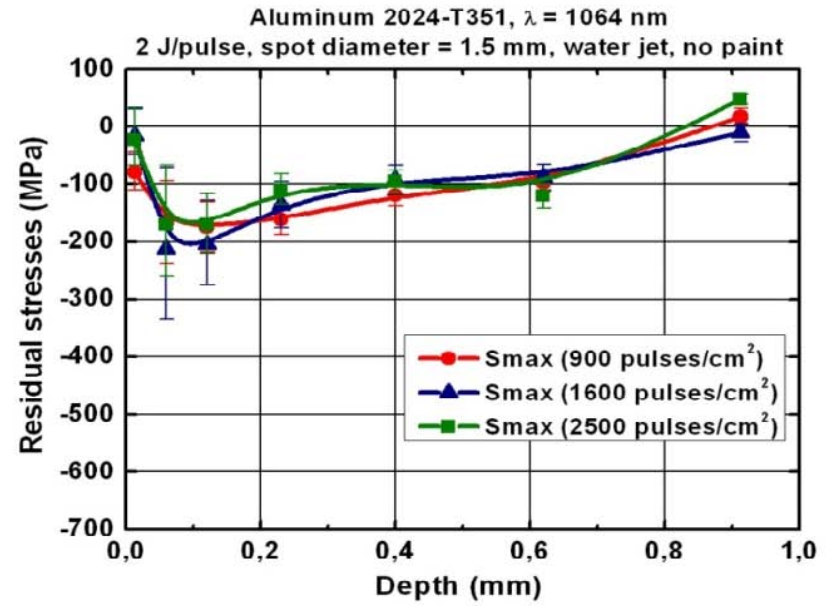

Fig. 6: Comparative analysis of induced Mohr maximum residual stresses profiles along z-coordinate in Al2024T351 for three representative pulse densities.

In both cases, the effective induction of compressive residual stress fields by the LSP treatment is observed, so that, in practice, in the optimum cases and taking into account that the reported values are minimum compressive stresses in absolute value, an effective protective field against 
crack aperture and propagation in depths up to near a millimeter can be assured.

Taking into account the respective values of Yield Strength of each of the considered materials $\left(\mathrm{YS}_{\mathrm{Al} 2024}\right.$ $=290 \mathrm{MPa}, \mathrm{YS}_{\text {Ti6Al4V }}=880 \mathrm{MPa}$ ), it can be observed that relatively important fractional values of preconstraint can be reached in both materials over relatively wide depths, a characteristic feature of LSP treatments that justifies its competitive character with other related existing surface treatment technologies, namely shot peening.

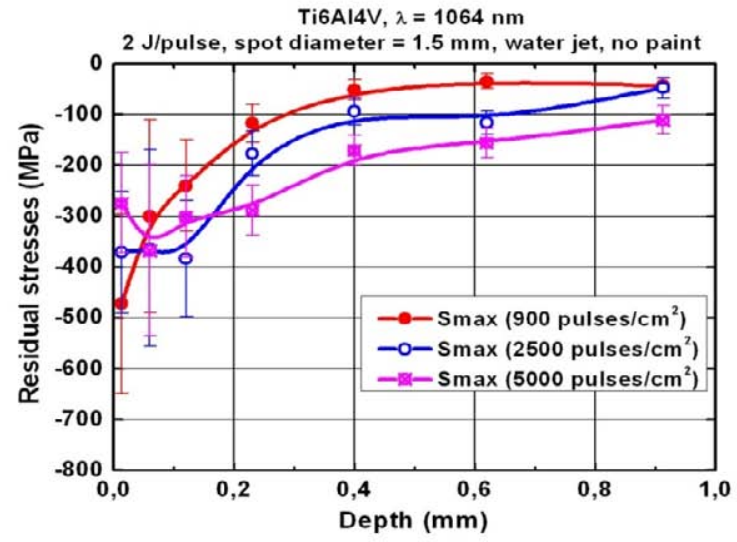

Fig. 7: Comparative analysis of induced Mohr maximum residual stresses profiles along z-coordinate in Ti6Al4V for three representative pulse densities.

\subsection{Induced Surface Roughness and Compactation}

In addition to residual stresses fields, surface roughness has been identified as having a significant influence on the degradation and fatigue behaviour of several critical components, especially due to corrosion and fretting fatigue [20-23].

A typical consequence of the application of the LSP technique at high EOD's is the generation on the treated surface of roughness patterns typically smoother than those of the original work-piece, a feature that has to be considered as highly favourable in comparison with the corresponding results obtained by surface treatments based on thermal processing or other mechanical treatments with comparatively lower intensity and overlapping factors, such as, typically, shot peening.

In Figs. 8 and 9, a sample is provided of the surface roughness profiles (obtained via Laser Confocal Microscopy) induced by the LSP technique on the two materials considered in the study for nearly-optimum EOD's.

In both cases a clear improvement of the material surface roughness can be appreciated in comparison with the untreated material, this modification being accompanied in the case of A12024-T351 (with a lower elastic limit) of a significant plastic deformation. In Table 1, the corresponding values of the plastically induced surface level compactation, $\langle\Delta \mathrm{z}\rangle$, and the $\mathrm{S}_{\mathrm{a}} 2 \mathrm{D}$ roughness parameter defined as $\mathrm{S}_{\mathrm{a}} \equiv \frac{1}{\mathrm{~A}} \iint_{\mathrm{A}}|\mathrm{z}(\mathrm{x}, \mathrm{y})-\overline{\mathrm{z}}| \mathrm{dA}$ are shown for different values of EOD in both materials.

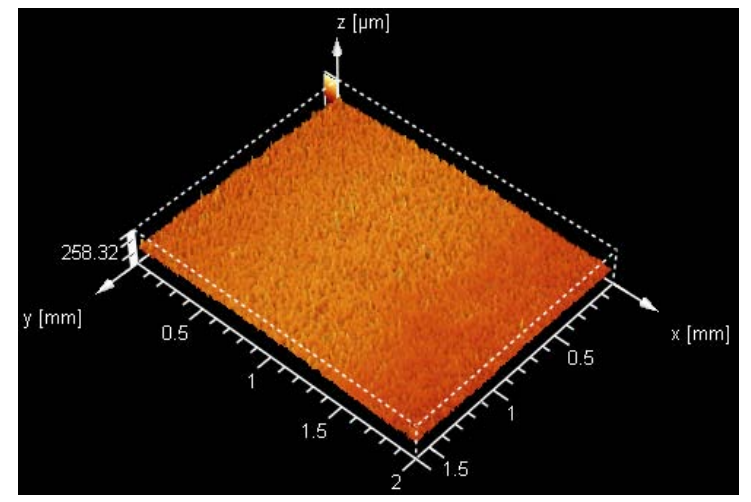

Fig. 8: Topographic Laser Confocal Microscope images showing the changes in surface roughness induced by the LSP technique in near-optimum conditions for Al2024.

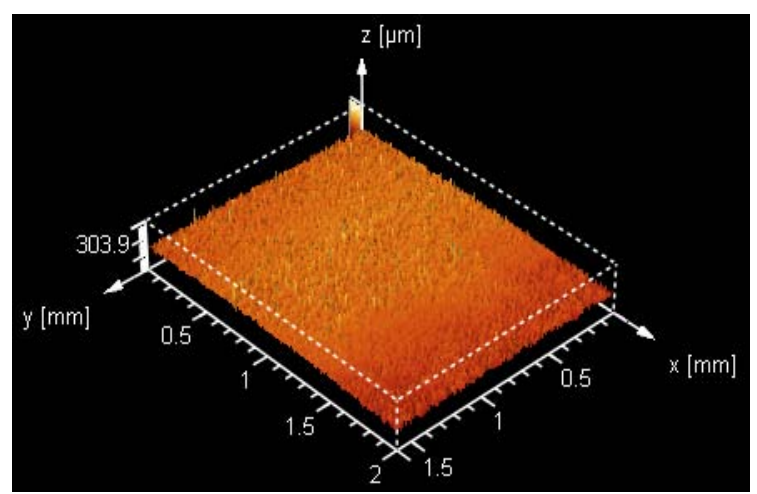

Fig. 9: Topographic Laser Confocal Microscope images showing the changes in surface roughness induced by the LSP technique in near-optimum conditions for Ti6Al4V.

Table 1: Summary of surface compactation results, $\langle\Delta z\rangle$, and $2 D$ surface roughness parameter $S a$ for different values of EOD in Al2024-T351 and Ti6Al4V.

\begin{tabular}{|c|c|c|c|c|c|}
\hline $\begin{array}{l}\text { Treatment } \\
\text { EOD } \\
\text { Surface } \\
\text { parameters }\end{array}$ & $\begin{array}{c}\text { No } \\
\text { Treat. }\end{array}$ & $\begin{array}{c}900 \\
\mathbf{p} / \mathbf{c m}^{2}\end{array}$ & $\begin{array}{l}1600 \\
\mathrm{p} / \mathrm{cm}^{2}\end{array}$ & $\begin{array}{l}2500 \\
\mathbf{p} / \mathbf{c m}^{2}\end{array}$ & $\begin{array}{l}\mathbf{5 0 0 0} \\
\mathbf{p} / \mathbf{c m}^{2}\end{array}$ \\
\hline $\begin{array}{c}<\Delta \mathrm{z}>(\mu \mathrm{m}) \\
\text { Al2024-T351 }\end{array}$ & ---- & 10.30 & 20.00 & 26.82 & \\
\hline $\begin{array}{c}\mathrm{S}_{\mathrm{a}}(\mu \mathrm{m}) \\
\text { Al2024-T351 } \\
\end{array}$ & 7.96 & 5.23 & 4.82 & 4.96 & \\
\hline $\begin{array}{c}<\Delta \mathrm{z}>(\mu \mathrm{m}) \\
\text { Ti6Al4V }\end{array}$ & ---- & 2.81 & 7.40 & & 5.80 \\
\hline $\begin{array}{l}S_{\mathrm{a}}(\mu \mathrm{m}) \\
\text { Ti6Al4V }\end{array}$ & 9.98 & 3.62 & 3.87 & & 3.87 \\
\hline
\end{tabular}


Additionally, in Fig. 10 (a and b), respective SEM transverse cuts of both treated materials in which the relative material compactation due to the LSP treatment can be clearly observed.
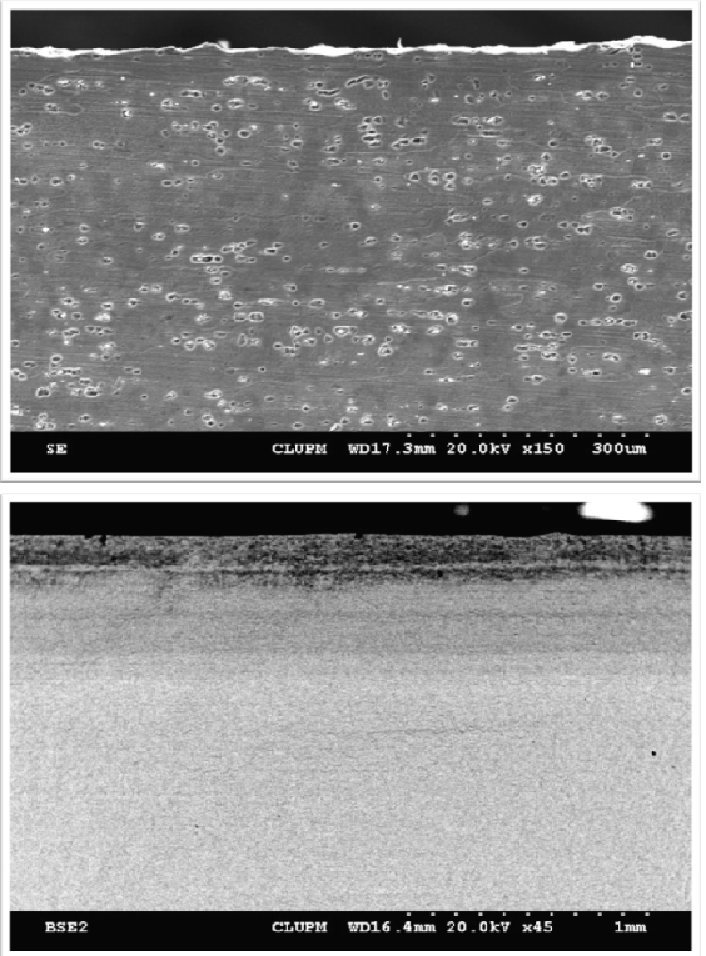

Fig. 10: SEM transverse cut of Al2024-T351 sample treated at 1600 pulses $/ \mathrm{cm}^{2}$ EOD and Ti6AlV sample treated with 5000 pulses $/ \mathrm{cm}^{2}$ EOD in which the compactation effect of the LSP treatment can be observed near the free surface.

On the basis of these results, the idea can be extracted of a rather significant surface smoothing provided by the LSP treatment, a key feature in view of the fatigue fretting and corrosion resistance of specimens treated by this technique, although the effect cannot, in general, be so directly controlled as the residual stresses fields by means of increasing treatment EOD's.

\subsection{Wear Resistance}

The just described results on surface roughness lowering by LSP have also a clear effect on the abrasive wear resistance of the treated specimens, although other possible surface transformation mechanisms (namely surface oxidation) finally determine the wear resistance of samples. In this case, materials able to experiment a physic-chemical transformation (carburization, nitriding, oxidation, etc.) can exhibit a differentiated behaviour concerning this property beyond the mechanical effects purely attributable to the LSP treatment.

As a clear example in this line, the comparative case of the two alloys considered in this paper (A12024-T351 and Ti6A14V) shows the difference between the wear behaviour of two crystalline phases developed by the LSP treatment in both materials with very different surface hardness properties, namely $\mathrm{Al}_{2} \mathrm{O}_{3}$ and $\mathrm{TiO}_{2}$, both appeared as a consequence of the surface heating resulting from the plasma generated by the incident laser beam. Although it has been shown by the authors that the effect of this plasma heating is not of a critical importance from the point of view of the residual stresses field obtained in the treated material after a certain depth [24], the effect of such heating seems to be important in what respects to hardness and wear properties.

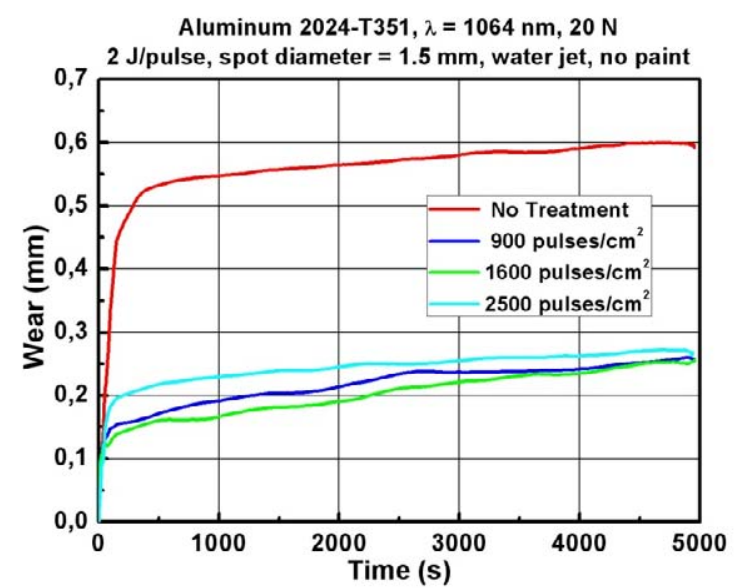

Fig. 11: Wear resistance results according to ASTM G9904 Standard of Al2024-T351 treated by LSP with different EOD's.

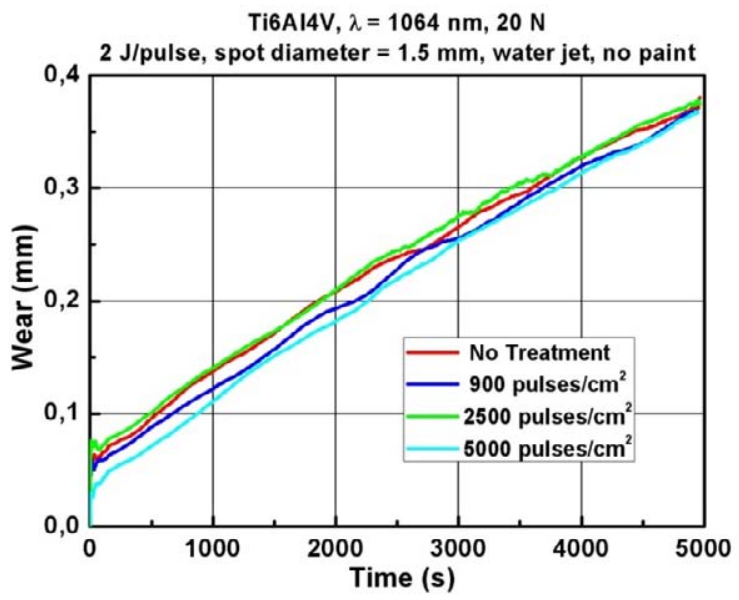

Fig. 12: Wear resistance results according to ASTM G9904 Standard of Ti6Al4Vtreated by LSP with different EOD's. 
In Figs. 11 and 12, the results of the wear resistance of the reference alloys for a $20 \mathrm{~N}$ load according to the ASTM G99-04 Standard are shown. It is clearly observed that, despite the slight variations induced in the friction coefficient as a consequence of the LSP treatment (responsible for the $2^{\text {nd }}$ order effects observed in the graphs, in general favourable to higher EOP LSP treatments), the appearance of a "hard" phase in A12024-T351 alloy following LSP treatment (that can be very clearly observed in SEM microstructure) and is not so hard in the case of Ti6A14V provides a physic-chemical mechanism for enhanced wear resistance.

\section{Conclusions}

In the present paper, a summary of surface effects induced by the LSP technique in two metallic alloys typical of the aerospace industry has been presented, a special focus on surface morphology and wear behaviour having been selected for this case.

Considering its primary interest for a broad range of mechanical properties susceptible to be enhanced by the LSP technique, a sample of the residual stresses depth profiles obtained in the considered materials for different treatment intensities (accounted for through the EOD parameter) has been primarily considered. The observation of these results clearly leads to the consideration of the LSP technique as really effective and controllable within wide limits for the induction of engineered residual stresses fields in critical components through modification of specific mechanical and chemical material properties, especially mechanical fatigue and corrosion resistance. The effect has been widely demonstrated by the authors in simple geometries and, with the aid of the developed calculational tools, can be readily applied to arbitrary component geometries.

Considering other important surface properties, an analysis has been presented of the effect of LSP on surface roughness, a relevant parameter conditioning the appearance of mechanical or corrosion failure at the surface level. In this case, LSP has been clearly shown to exert a positive effect on the surface evenness, presumably beneficial concerning specifically fatigue fretting and pitting corrosion.

Finally, the analysis of wear resistance of specimens of both materials treated by the LSP technique has been reported, the important fact having being found of the significant influence of the ambient processing conditions on the final mechanical performance of the treated surface. Concretely, the relatively important influence of possible oxidation or other physic-chemical surface modification mechanisms in the case of alloys presenting the appropriate elemental composition has been reported.

This fact has to be considered of a high relevance in view of its inherent difficulty to be adequately controlled. Both the control of atmospheric treatment conditions and the dynamics of the laser produced plasma near the material surface are key issues deserving a further detailed evaluation.

From the point of view of process design, the practical implementation of LSP processes involves the consideration of multiple features, the most critical of which is considered to be the uniformity of the residual stresses fields after the treatment.

Trying to involve the different factors influencing the final quality and performance of the treatment, two parameters additional to the studied EOD have been defined, namely:

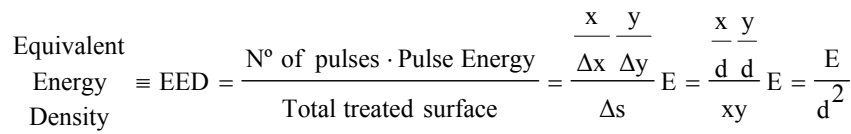

$$
\begin{aligned}
& \underset{\text { Overlapping Factor }}{\text { Equivalent Local }} \equiv \mathrm{ELOF}=\frac{\mathrm{N}^{\mathrm{o}} \text { of pulses } \cdot \text { Pulse Area }}{\text { Total treated surface }}=\frac{\frac{\pi}{4} \phi^{2}}{\mathrm{~d}^{2}}=\frac{\pi}{4}\left(\frac{\phi}{\mathrm{d}}\right)^{2}
\end{aligned}
$$

According to the high values used for the ELOF parameter, this condition is widely fulfilled in all the treatment reported in this paper, although a degree of liberty is thought to clearly exists in selecting the related EOD parameter for equivalently homogeneous treatments.

However, the selection of lower EOD's leads, as clearly shown in the reported figures, to either less intense or shallower residual stresses fields into the piece (the second important feature to be born in mind for the design of LSP treatments), what finally means a poorer degree of protection for the corresponding component. As suggested by the definition of the EED parameter, a proper combination of the ELOF and EOD parameters can be achieved through the modulation of the applied pulse energy, E, an issue presently under study.

As a final remark concerning the practical significance of the LSP technique, it is considered that the life cycle improvement achievable by the application of the treatment to specific high reliability components in different sectors as, primarily, aerospace, biomedical and nuclear, has an evident positive incidence on their long-term ecological balance, so that the technique has to be considered as a sustainability-supporting one. 


\section{Acknowledgement}

Work supported by MEC/MCINN (Spain; Projects DPI2005-09152-C02-01; MAT2008-02704/MAT) and EADS-CASA (Spain)

\section{References}

[1] Fairand, B.P. et al.: J. Appl. Phys., $\underline{43}$, 3893-3896 (1997).

[2] Yang, L.C.: J. Appl. Phys., 45, 2601-2607 (1974)

[3] Fairand, B.P., Clauer, A.H.: J. Appl. Phys., $\underline{50}$, 1497-1502 (1979).

[4] Sano, Y. et al.: "Underwater Laser Processing to Improve Residual Stress on Metal Surface". In Proceedings of the 6th International Welding Symposium of Japan Welding Society, Nagoya, Japan, 501-506 (1996).

[5] Dane, C.B.: "High Power Laser for Peening of Metals Enabling Production Technology". In Lawrence Livermore National Laboratory, Report UCRL-JC-131104-Rev 1 (1998).

[6] Sano, Y. et al.: Nucl. Inst. Meth. Phys. Res. B, 121, 432-436 (1997).

[7] Nikiforov, Y. et al.: Mat. Sci. Eng. A $\underline{288}, 173-176$ (2000).

[8] Peyre, P. et al.: J. Mat. Sci., $\underline{33}$, 1421-1429 (1998).

[9] Akita, K. et al.: Mat. Sci. Forum, 490-491, 370-375 (2005).

[10] Ocaña, J.L. et al.: "A Model for the Coupled Predictive Assessment of Plasma Expansion and Material Compression in Laser Shock Processing Applications". In High-Power Laser Ablation II, Phipps, C.R., Niino, M., Eds., SPIE Proceedings, Vol. 3885, 252-263 (2000).

[11] Ocaña, J.L. et al.: "Predictive assessment and experimental characterization of the influence of irradiation parameters on surface deformation and residual stresses in laser shock processed metallic alloys". In High-Power Laser Ablation V, Phipps C.R., Ed.. SPIE Proceedings, Vol. 5548, 642-653 (2004)

[12] Ocaña, J.L. et al.: "A review of the physics and technological issues of high intensity laser shock processing of materials as a method for mechanical properties modification". In XVI International Symposium on Gas Flow, Chemical Lasers and High-Power Lasers, Schuöcker, D., Ed. SPIE Vol. 6346, 63461P, (2006).

[13] Morales, M. et al.: Mat. Sci. Forum, $\underline{638}-\underline{642}, 2682-$ 2687 (2010).

[14] Colvin, J.D. et al.: Physics of Plasmas, 10, 29402947 (2003).

[15] Zhang, W., Yao, Y.L.: J. Manufacturing Sci. Eng., 124, 369-378 (2002).

[16] MacFarlane, J. J. et al.: J. Quant. Spectrosc. Radiat. Transfer, 99, 381-397 (2006).
[17] Ocaña, J.L. et al.: Appl. Surf. Sci., 238, 501-505 (2004).

[18] ASTM International: "ASTM E837-08 Standard Test Method for Determining Residual Stresses by the Hole-Drilling Strain Gage Method" (2008).

[19] ASTM International 2004: "ASTM G 99-04 Standard Test Method for Wear Testing with a Pin on Disk Apparatus" (2004).

[20] Ocaña, J.L. et al.: "Laser Shock Processing as a Method for Surface Properties Modification of Metallic Materials". In Shot Peening and other Mechanical Surface Treatments. V. Schulze, A. Niku-Lari, Eds. I.I.T.T. Paris, 466-471 (2005).

[21] Sánchez-Santana, U. et al.: Wear, 260, 847-854 (2006).

[22] Rubio-González, C. et al.: Appl. Surf. Sci., 252, 6201-6205 (2006).

[23] Grum, J. et al.: Mat. Sci. Forum, 537-538, 655-662 (2007).

[24] Ocaña, J.L. et al.: Mat. Sci. Forum, 539-543, 11161121 (2007). 